\title{
OPUCEM: A Library with Error Checking Mechanism for Computing Oblique Parameters
}

\author{
Özgür Çobanoğlu ${ }^{a}$, Erkcan Özcan $^{b}$, Saleh Sultansoy $^{c}$ and Gökhan Ünel ${ }^{d}$
}

November 4, 2018

\begin{abstract}
${ }^{a}$ European Nuclear Research Center, CERN, Geneva, Switzerland. ${ }^{b}$ Department of Physics and Astronomy, University College London, London, UK. ${ }^{c}$ TOBB ETU, Physics Department, Ankara, Turkey and Institute of Physics, Academy of Sciences, Baku, Azerbaijan. ${ }^{d}$ Physics and Astronomy Department, University of California at Irvine, Irvine, USA.
\end{abstract}

\begin{abstract}
After a brief review of the electroweak radiative corrections to gauge-boson self-energies, otherwise known as the direct and oblique corrections, a tool for calculation of the oblique parameters is presented. This tool, named OPUCEM, brings together formulas from multiple physics models and provides an error-checking machinery to improve reliability of numerical results. It also sets a novel example for an "open-formula" concept, which is an attempt to improve the reliability and reproducibility of computations in scientific publications by encouraging the authors to open-source their numerical calculation programs. Finally, we demonstrate the use of OPUCEM in two detailed case studies related to the fourth Standard Model family. The first is a generic fourth family study to find relations between the parameters compatible with the EW precision data and the second is the particular study of the Flavor Democracy predictions for both Dirac and Majorana-type neutrinos.
\end{abstract}

\section{Introduction}

The categorization of the electroweak (EW) corrections based on their contribution types dates back to a study of photon propagated four-fermion processes [1]. The corrections to vertices, box diagrams and bremsstrahlung diagrams were all considered as "Direct" whereas the propagator corrections due to vacuum polarization effects were all named as "Oblique" since these participate to the computations in an indirect manner [2].

The EW precision data collected over the last few decades by various particle physics experiments have often been used to constrain many new models of particle interactions. They are particularly useful in checking the allowed parameter space of a given model through its contributions especially to the vacuum polarization corrections to the boson propagators. The main oblique parameters are usually denoted by letters $S$, $T, U$ and the auxiliaries with letters $V, W, Y$ [3]. As an example, the $S$ parameter estimates the size of the new fermion sector and the $T$ parameter measures the isospin symmetry violation, i.e. the split between the masses of the new up and down-type fermions. The Standard Model is defined by the values $S=T=U=0$ for a given top quark and Higgs boson mass.

Together with the detailed review by Peskin and Takeuchi [2], a number of papers were published, calculating the contri- bution of a given model to the oblique parameters. To name a few, the estimation of the number of fermion families and neutral gauge bosons [4], the validity consideration of the Higgsless models [5], and the investigation of the Majorana nature of the neutrinos [6] can be cited. However, a number of such publications suffer from unusual notations with typos in formulas, and errors arising due to utilization of approximations instead of exact calculations (with assumptions such as $m_{H}>>m_{Z}$ ) or in some cases from unguarded remarks such as "heavy" for the new fermions.

The goal of this work is two fold: the first is to present a library to compute the oblique parameters $S, T$ and $U$ both with exact one-loop calculations and with some well-defined approximations for a number of models and the second is to scan the available parameter space for the fourth family models. The comparisons between exact and approximate computations, and amongst formulas from different papers provide an error checking machinery which improves the enduser reliability. Implemented in $\mathrm{C} / \mathrm{C}++$ languages, we call this library OPUCEM, which stands for Oblique Parameters Using $\mathrm{C}$ with Error-checking Machinery. The OPUCEM package consisting of the library and a set of example driver and presentation functions, which are discussed in this manuscript, are publicly available [7].

The next section describes the technical details of the library implementation. Then the following sections are on detailed physics studies demonstrating the use cases of OPUCEM. Section 3 uses the OPUCEM library to investigate the plausibiliy of a generic fourth Standard Model (SM) family (SM4). Section 4 focuses on the implications of the EW data from the viewpoint of the flavor democracy (FD) hypothesis, which provides a principle theoretical motivation for the potential existence of a fourth SM family. These case studies deal with defining the parameter regions favored by the data and define a set of benchmarking points for the SM4. In both sections, Dirac and Majorana cases of the fourth SM family neutrino are investigated separately. Finally in Section 5, we present our concluding remarks.

\section{Function Library Implementation}

The OPUCEM library mainly consists of a header file opucem.h declaring the available function prototypes and opucem.c implementing them. The functions are grouped by the relevant physics cases such as Majorana neutrinos or Higgs bosons. In each case, internal comments are used to document the source code, indicating the reference paper for each of the formula and the nature of the calculations (e.g. exact 1-loop calculations, or approximations valid under certain assumptions such as the new fermions being much heavier than the Zboson). Compilation of the library is straightforward, however a makefile is provided as is customary. The makefile also features additional targets to produce example command-line and graphical-user-interface applications that make use of the library in studying the fermions of a fourth SM family [8, 9, 10].

One of the primary goals of the implementation is portabil- 
ity, since we consider portability an auxiliary measure of the reliability of the code. To facilitate this goal, the initial implementation was done in pure $\mathrm{C}$, with the code having no dependencies or requirements beyond a standards-compliant $\mathrm{C}$ compiler. Since certain formulas make use of complex numbers, we made use of the complex type found in the C99 standard. However, starting with version 00-00-03, the C99 complex type was dropped in favor of the std::complex template in the $\mathrm{C}++$ standard template library. The main motivation for this change was to provide an easy roadmap for an eventual replacement of various long double variables with multiprecision variables.

The compilation and execution is compatible with any UNIX-like environment with modern versions of the GNU compiler collection (gcc) [11], that support a subset of the complex functions given in the C99 standard. For example, the last version which uses the pure $\mathrm{C}$ implementation (v0000-02) is known to compile with gcc 4.x. On the other hand, the current version (v00-00-04), which makes use of the C++ std::complex templates, can only be compiled with versions of g++ 4.2 onwards, which implements the part of the C++ Technical Report 1 (TR1) that pertains to complex numbers. TR1 introduced the complex inverse hyperbolic functions of C99 into the std::complex template, and these additions are expected to appear in the upcoming $\mathrm{C}++0 \mathrm{x}$ standard as well.

While we do not intend to support Microsoft Windows operating system as a regular OPUCEM target, we still have ported the $\mathrm{C}$ version of the library using the GNU development toolchain provided under the Cygwin environment and independently by the native tools from the MinGW project $[12,13]$. The main missing ingredients are complex numbers with long double precision. The latest MinGW distribution (MinGW 5.1.6) comes with complex.h implementations for gec 3.4.4 and gec 4.3.4 but only with double precision. For this reason, for the Windows port, we rely on an external implementation of the C99 complex numbers implemented by S. Moshier [14]. It should be noted that this complex-number implementation, along with the long double math library from the same author, makes it possible to port the $\mathrm{C}$ version of OPUCEM to any architecture that supports even rather old versions of the GNU Compiler Collection. (For example under Cygwin 1.5.25, we were able to compile even with gcc 3.4.4.) Under a separate Windows branch, we provide documentation and makefiles to facilitate such efforts.

Finally it should be mentioned that the current version of the code, while using the $\mathrm{C}++$ complex templates, is still structured in a strictly $\mathrm{C}$-like manner, making no use of the objectoriented features of $\mathrm{C}++$. Because of this, it is clear that the enduser experience is likely to quickly deteriorate as more and more physics models are added to the library in the future. Therefore for the upcoming revisions of the code, the planned updates include a better $\mathrm{C}++$ implementation, in particular making use of namespaces to separate formulas from different physics models.

The oblique parameter calculation is implemented for the following scenarios in the current version of the OPUCEM library:

- New lepton doublets with Dirac [15] or Majorana [16] type neutral leptons;

- New quark doublets with variable $4 \times 4 \mathrm{CKM}$ mixing to the third generation [17];

- SM and 2 Higgs Doublet Model (2HDM) Higgs bosons $[18,15]$.

Additionally, a set of functions for recalculation of the SM origin in the $S-T$ plane based on reference values of the top quark and the Higgs boson masses is also available.
During the implementation of the library, a number of minor errors on the reference papers have been found and corrected. These are:

- mixing angle in the definition of the left-handed weak eigenstates of the two Majorana neutrinos in [16]; their equation 10 should read $c_{\theta}^{2}=M_{2} /\left(M_{1}+M_{2}\right), \quad s_{\theta}^{2}=$ $M_{1} /\left(M_{1}+M_{2}\right)$.

- contribution to $\mathrm{W}$ and $\mathrm{Z}$ boson self energies induced by the two Majorana neutrinos in [6]; the right hand side of their equation 3.6 should start as $\ldots=\frac{1}{12 \pi^{2}}\left[\left[q^{2}-\frac{m_{1}^{2}-m_{2}^{2}}{2} \pm\right.\right.$ ... (See the corrected form in [19]).

\subsection{The error-checking mechanism}

To ensure the correct calculation of the oblique parameters, a number of validation techniques have been used. The core part of the library has been pair programmed, with subsequent updates consistently cross-checked against the earlier parts. Furthermore, a number of sanity checks have been performed during the implementation. Some of these checks are documented as embedded comments in the source code. For example, by setting the masses of the second doublet Higgs particles in the 2-Higgs-Doublet-Model to zero and taking into account the extra multiplicative factor of 3 , we have verified the calculation of the contribution from the SM Higgs boson. Other sanity checks have been promoted to become actual run-time tests, accessible through clearly indicated safe-to-use functions. For example, the contributions to $S$ and $U$ parameters from Majorana-type neutrinos requires high precision, for which the long double type might not be adequate under certain values of the input parameters. To catch such cases automatically, we check whether the computations for Majoranatype neutrinos converge to those of the Dirac-type in the fullydegenerate limit of the two Majorana masses. Moreover, these safe-to-use functions compare approximate and exact computations and display warnings if absolute or relative difference between the two are above certain predefined thresholds. Finally, special cases such as equal mass values for up and down type fermions, have been addressed carefully with limiting cases of the formulas derived using symbolic computation tools.

We have also tried to make sure that the OPUCEM implementation of the formulas can correctly reproduce the tables and plots in the previously published work. An example of this has been available in the examples/prd48_pg225 directory since the very first public version of the library. The provided shell script generates an executable that calculates $S$ and $U$ as a function of the lepton mass parameter $\xi$, reproducing the first figure of [6], as shown in Figure 1.

\subsection{Additional tools and presentation functions}

It is worth noting that some of the comparisons mentioned in the previous section cannot go beyond being qualitative analyses, due to the fact that quantitative information is missing in the original publications. In such cases, we have tried to manually extract values from the published figures and documented the level of agreement as auxiliary notes spread as comments in the source code. In a few cases, we have also discovered that the representation of data in the figures was incorrect.

An example of such a mistake is in the Figure 2 of [20], where the $U=0$ fit results to the $\mathrm{EW}$ precision data are being plotted on the $S-T$ plane. In that figure, the $90 \%$ confidence level (CL) ellipse seems to have been plotted instead of the 2 -sigma (95\% CL) error ellipse. In our Figure 2, we show both the $90 \%$ CL and 95\% CL error ellipses to highlight the enlarged parameter space. In the same paper, we have also identified additional minor errors in the value of the $T$ parameter for $m_{H}=300 \mathrm{GeV}$ and in the position of the $S-T$ origin, 

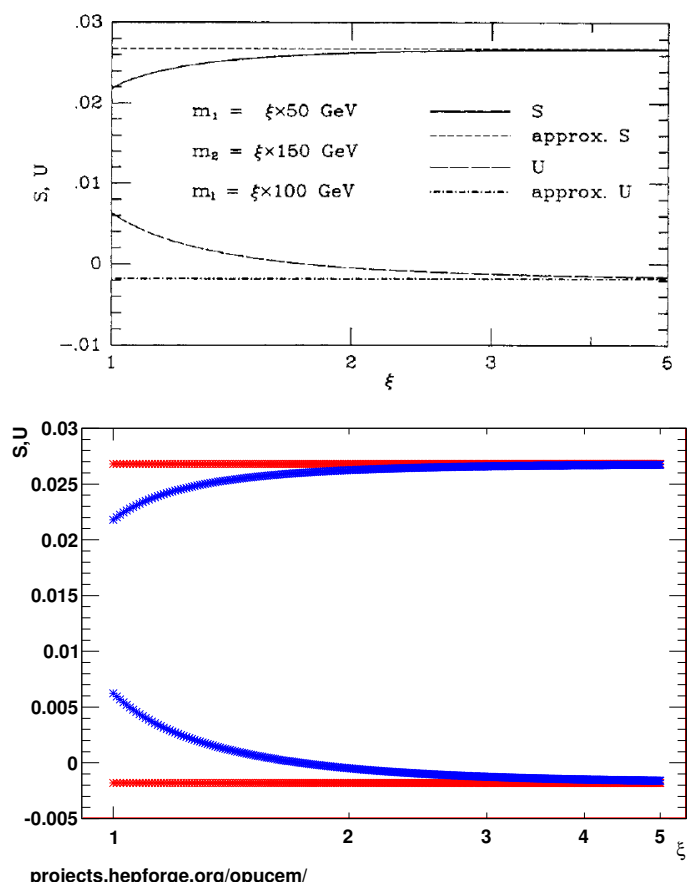

Figure 1: Contributions to $\mathrm{S}$ and $\mathrm{U}$ due to fourth-generation leptons, $N_{1}, N_{2}$ and $\ell$, with masses $m_{1}=\xi \times 50 \mathrm{GeV}, m_{2}=$ $\xi \times 150 \mathrm{GeV}$ and $m_{\ell}=\xi \times 100 \mathrm{GeV}$ as a function of $\xi$. The top plot is from [6] and the bottom plot is the output of OPUCEM library. In both cases exact and approximate calculations are compared.

after their recomputation to take into account new reference values of the the top quark and presumed Higgs boson masses. Although the relative size of all these errors are rather small $(\sim 3-4 \%)$, and the impact on the conclusions of the paper is negligible, we mention them here for the sake of accuracy.

In order to facilitate correct representation of the EW data on the $S-T$ plane, we provide our plotting routines as part of the OPUCEM package, under the tools/STellipse directory. Error ellipses can easily be drawn from the central values and the uncertainties of the measured $S, T$ values and their correlation coefficient. These plotting functions make use of the ROOT package [21], an object-oriented $\mathrm{C}++$ analysis framework from CERN and the de facto standard for statistical analysis in the experimental high-energy physics community today.

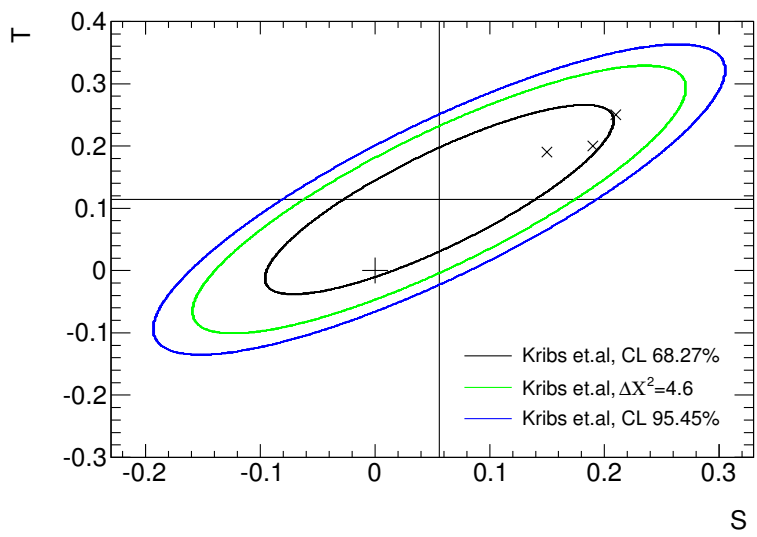

Figure 2: Corrected Figure 2 from Kribs et al. [20]

\subsection{Graphical User Interface}

Besides the full-featured standard command-line, the package also provides users with a light-weight graphical user interface (GUI) for demonstration purposes. The GUI developed within the ROOT [21] framework, is actually an independent application loading the OPUCEM libraries at run-time. It makes relevant function calls based on the user set of inputs and simply displays the results accordingly on the $S-T$ plane.

As it can be seen on the Figure 3, the GUI provides simple input boxes for the input parameters such as the fermion masses and also access to the internal parameters used in the calculations such as the sine squared of the weak mixing angle. The user can perform the $S-T$ calculation, see the resulting data points being plotted and save the plot in multiple formats (e.g. eps, png, root, etc.) using the buttons at the bottom of the GUI window. For example, majority of the $S-T$ plots shown in this manuscript have been generated using the OPUCEM GUI.

Like the library itself, the OPUCEM GUI has been tested on various operating systems (OS) running different ROOT releases. These include but are not limited to SLC-5, Mac OSX 10.5 and 10.6 running ROOTv5.26.00(b), Ubuntu-9.10 and 10.4 running ROOTv5.18.00b. However it should be noted that while the GUI can both be interpreted in ROOT and be natively compiled, the library functions themselves are always loaded dynamically in binary form at run time.

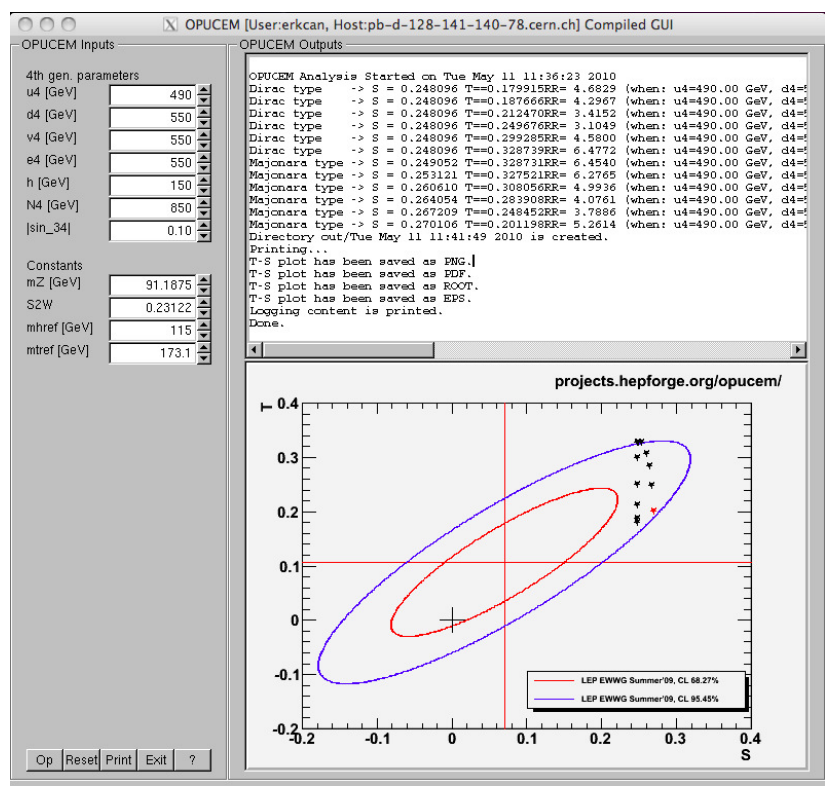

Figure 3: The OPUCEM graphical user interface.

We have chosen the ROOT framework for visualization and GUI development as an example, however we do not necesarily endorse any specific framework. Since OPUCEM is a generic function library, it can be loaded and instantly be used from within any rapid application development (RAD) environment such as MONO[22] and GAMBAS[23] for Basic, QtCreator[24] for $\mathrm{C} / \mathrm{C}++$, and potentially MS Visual Studio for .NET pretty much the same way. This makes possible applications of the OPUCEM library available on almost any platform.

\section{The fourth SM family in General}

For the rest of this paper, we make use of OPUCEM to study the Standard Model with 4 generations of fermions (SM4) in order to determine the parameter space compatible with the precision EW data and to look for possible correlations among the SM4 parameters. A fourth SM family, with fermion doublets of masses heavier than $100 \mathrm{GeV}$, could explain some 
emerging new physics hints from current particle physics experiments and also cure some of the theoretical shortcomings of the SM itself $[8,9,10]$. The compatibility of the additional generations with the electroweak data was previously investigated using a multi-parameter fit approach [25, 17].

\section{1 $S, T$ Formalism and SM4}

Before using OPUCEM to scan the fourth generation parameter space, we would like to point to the excellent applicability of the $S, T$ formalism in studies of SM4, by providing an example comparing the results from a global EW fit (involving 16 input parameters and more than 10 degrees of freedom) to those obtained from the oblique parameters. In a recent publication, it has been demonstated that the mixing between the third and the fourth generation quarks, $\theta_{34}$, plays an important role in the determination of the best fit to the EW precision data [17]. A resulting plot from this publication is reproduced in Figure $4 \mathrm{a}$, in which the most probable value of the Higgs boson mass obtained from the global fit is plotted as a function of $\left|\sin \theta_{34}\right|$. OPUCEM library is able to closely reproduce this result without a global fit by scanning the $m_{H^{-}}\left|\sin \theta_{34}\right|$ parameter space, as shown in Figure 4b. The different colors correspond to different $\Delta \chi^{2}$-probability values. There is a very good agreement between the most possible values obtained from OPUCEM and the trend of the global fit results from [17].

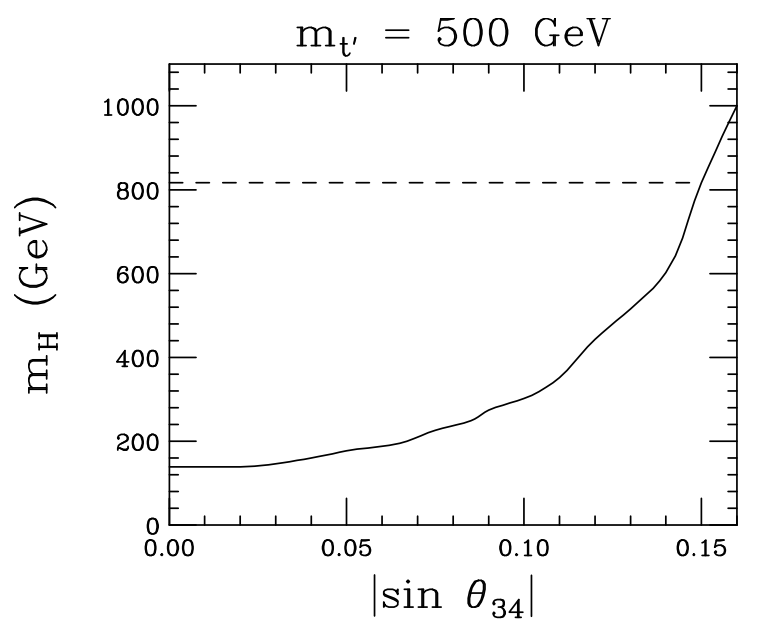

(a)

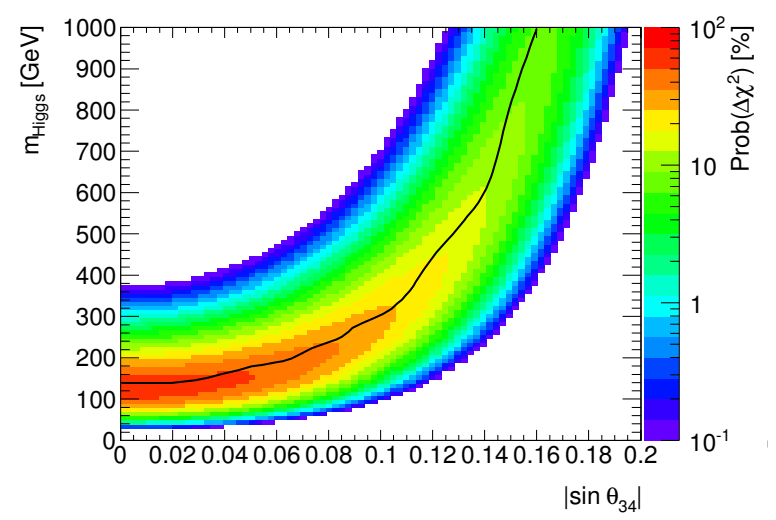

(b)

Figure 4: Higgs mass as a function of the mixing angle between 3rd and 4th generations. (a) Results of the global fit from [17]. (b) $\Delta \chi^{2}$ probability obtained from a scan of the two parameters as obtained using OPUCEM. The 4th generation fermion masses are taken to be $m_{e_{4}}=145 \mathrm{GeV}, m_{v_{4}}=$ $100 \mathrm{GeV}, m_{u_{4}}=500 \mathrm{GeV}, m_{d_{4}}=445 \mathrm{GeV}$. The curve from plot (a) has been reproduced on plot (b) for easy comparison.

\subsection{Scanning SM4 with OPUCEM}

Neglecting possible mixings in the fermionic sector as motivated by the preference for small $\left|\sin \theta_{34}\right|$, one deals with 5 parameters (masses of the four new fermions, $u_{4}, d_{4}, e_{4}, v_{4}$, and of the Higgs boson) if the neutrino is of Dirac type like the other fermions. However in the realm of the SM, there is no reason to forbid the Majorana mass terms for righthanded components of neutrinos. In this case, the neutrino transforms into two Majorana particles. We will denote the lighter of these as $v_{4}$ and the heavier as $N_{4}$. Thus in the Majorana case, one has an extra mass parameter $\left(m_{N_{4}}\right)$ to consider. Given the many degrees of freedom, we initially follow a simple scanning method: namely, we choose definite value(s) for Dirac(Majorana) neutrino mass(es) and scan the masses of the three charged fermions and the Higgs boson. We note that similar scans with more details have very recently appeared in the literature, but only for Dirac-type neutrinos [26, 27].
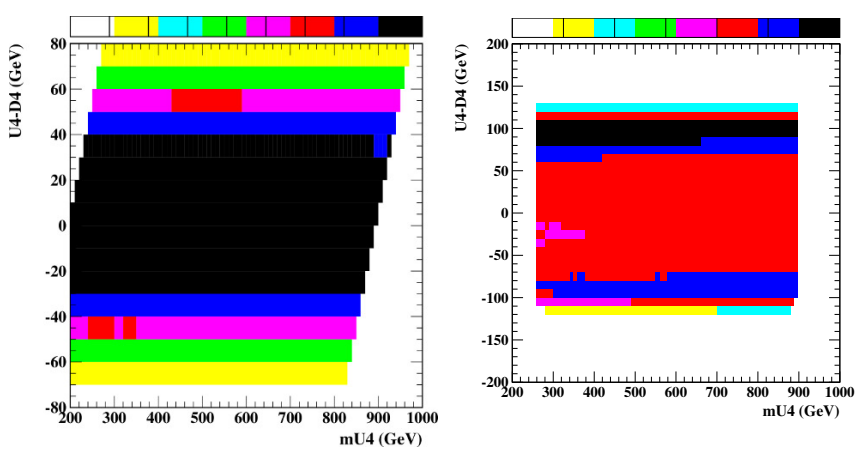

Figure 5: The correlation between the fourth family quark masses and mass differences for Dirac (left) and Majorana (right) cases.

We start by an analysis of the quark sector. The results are presented in Figure 5 for Dirac (left) and Majorana (right) cases. The mass values, for which the computed $S$ and $T$ fall within the $2 \sigma$ error ellipse of the LEP EW working group's $U=0$ fit [28], are accepted. Darker regions correspond to more entries, whereas the white region has no entries. We scan the charged fermion masses from $200 \mathrm{GeV}$ to $1 \mathrm{TeV}$ and Higgs mass from $115 \mathrm{GeV}$ to $900 \mathrm{GeV}$. In the Dirac neutrino case, the neutrino mass is assumed to be, $m_{v_{4}}=90 \mathrm{GeV}$, the lowest value allowed by the LEP data [29]. It is seen that the preferable values for $u_{4}-d_{4}$ mass difference lie between $30 \mathrm{GeV}$ and $+40 \mathrm{GeV}$, independent of the quark mass. However, values between $-70 \mathrm{GeV}$ and $+80 \mathrm{GeV}$ are also allowed. Studying other neutrino mass values, we observe that these results show a slight dependence on the fourth neutrino Dirac mass. In the Majorana neutrino case, we initially assume $m_{v_{4}}=80 \mathrm{GeV}$, the lowest value allowed by the LEP data [29] and $m_{N_{4}}=1000 \mathrm{GeV}$. It is seen that although the preferable values for $u_{4}-d_{4}$ mass difference lie around $100 \mathrm{GeV}$, other values between $-110 \mathrm{GeV}$ and $+130 \mathrm{GeV}$ are also allowed. If the lighter neutrino mass increases, the favoured $u_{4}-d_{4}$ mass difference also increases, as an example for $m_{v_{4}}=280 \mathrm{GeV}$, the preferred $u_{4}-d_{4}$ mass difference lies around $170 \mathrm{GeV}$.

In Figure 6, the correlation between the fourth family lepton mass and quark mass differences are shown for Dirac (left) and Majorana (right) neutrinos. In this figure, where the darker (lighter) regions correspond to more (less) entries, only the mass values falling into the $2 \sigma$ error ellipse of $S$ and $T$ parameters are shown. The charged fermion masses from $200 \mathrm{GeV}$ to $1000 \mathrm{GeV}$ and Higgs mass from $115 \mathrm{GeV}$ to $900 \mathrm{GeV}$ have been scanned. Neutrino masses are selected as in Figure 5.

It is seen that only $e_{4}$ masses below 275 (390) GeV are allowed for Dirac (Majorana) cases for a $u_{4}-d_{4}$ mass difference that lie between $-80 \mathrm{GeV}$ and $+90 \mathrm{GeV}$, independent of the quark mass. 

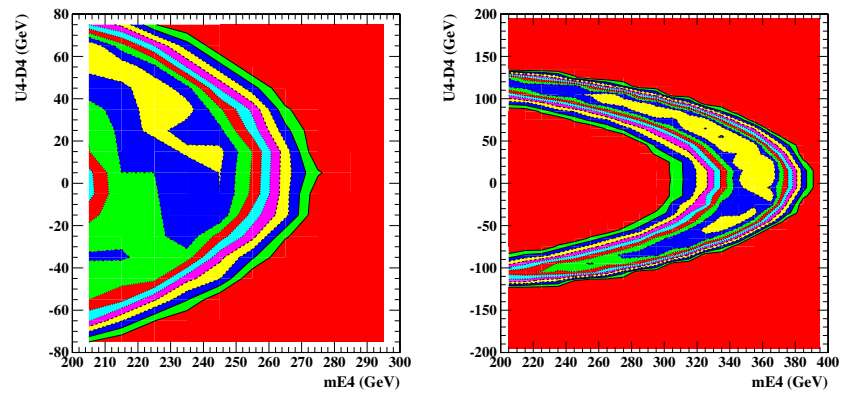

Figure 6: The correlation between the fourth family lepton mass and quark mass differences and for Dirac (left) and Majorana (right) cases.

\subsection{Higgs Mass Dependence}

The impact of the Higgs mass is better understood with a reduced set of parameters. To that end, and based on the hint from the mass difference studies, the correlation of the mass difference between the quark sector and the lepton sector is studied for different values of the Higgs boson mass. However, in this study, the fermion masses themselves are not used as independent variables; while keeping the sum of the quark and lepton masses as equal and constant, the probabilities are calculated as a function of $u_{4}-d_{4}$ and $e_{4}-v_{4}$ mass difference. The upper part of Figure 7 shows the $\chi^{2}$ probability of fourth generation's compatibility with the EW precision data for $m_{H}=600 \mathrm{GeV}$ but for different values of the fermion mass sums: from left to right $m_{u_{4}}+m_{d_{4}}=m_{e_{4}}+m_{v_{4}}=$ 600,1000 and $1600 \mathrm{GeV}$. Different colors correspond to different probabilities with black being the highest and blue the lowest as given in the scale on the right hand side. The most probable mass differences constitute an ellipse in the $m_{u_{4}}-m_{d_{4}}, m_{e_{4}}-m_{v_{4}}$ plane, independent of the fermion mass sums. The ellipse obtained from the fit to the $1600 \mathrm{GeV}$ results, can be seen on all 3 plots in yellow solid line. The impact of the Higgs boson mass is shown in the lower 3 plots, where the fermion mass sum is taken as $1600 \mathrm{GeV}$ but the Higgs boson mass changes as 115,300 and $600 \mathrm{GeV}$, from left to right. One can notice that as the Higgs boson mass increases, so do the fitted ellipse's radii.

The results of the fitted ellipse's semi-minor axis length as a function of $\ln \left(m_{H}\right)$ are given in Figure 8, the upper plot. The lower plot shows that the semi-major axis is always 1.75 times longer than its semi-minor axis. It is therefore possible to summarize the results and parameterize the core of the valid region as a function of the Higgs mass (all masses in $\mathrm{GeV}$ ):

$$
\begin{aligned}
x & \equiv m_{e_{4}}-m_{v_{4}} \\
y & \equiv m_{u_{4}}-m_{d_{4}} \\
r & =-31.11+19.3 \ln \left(m_{H}\right) \\
1 & =\frac{x^{2}}{r^{2}}+\frac{y^{2}}{(1.75 r)^{2}}
\end{aligned}
$$

For Majorana-type neutrinos, the compatibility of the fourth generation with EW data depends also on the heavier Majorana neutrino's mass. This dependency can be illustrated using three example scenarios with different Higgs boson mass values, shown with open circles in Figure 9. The open circle inside the 1-sigma ellipse corresponds to $m_{H}=115 \mathrm{GeV}$ and $m_{u_{4}}=m_{d_{4}}=300 \mathrm{GeV}, m_{v_{4}}=245 \mathrm{GeV}$ and $m_{e_{4}}=355 \mathrm{GeV}$. The open circle inside the 2-sigma ellipse corresponds to a higher Higgs boson mass, of $450 \mathrm{GeV}$ and to fermion masses as $m_{u_{4}}=335 \mathrm{GeV}, m_{d_{4}}=m_{v_{4}}=265 \mathrm{GeV}$ and $m_{e_{4}}=335 \mathrm{GeV}$. The third open circle, the farthest from the center of the $S-T$ minimum is for the heaviest Higgs mass: $900 \mathrm{GeV}$ with the correspond-
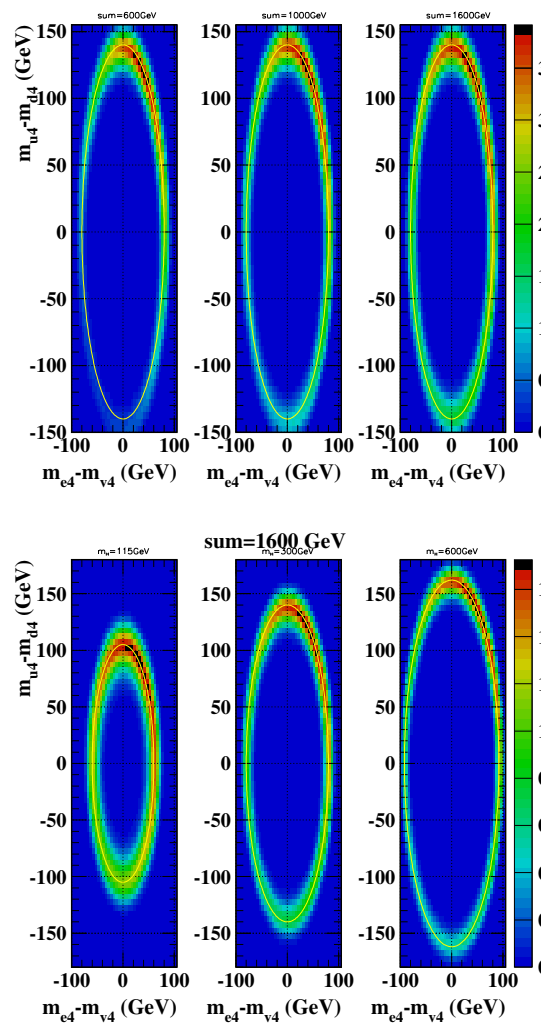

Figure 7: The correlation between the fourth family lepton mass and quark mass differences for Dirac neutrinos.
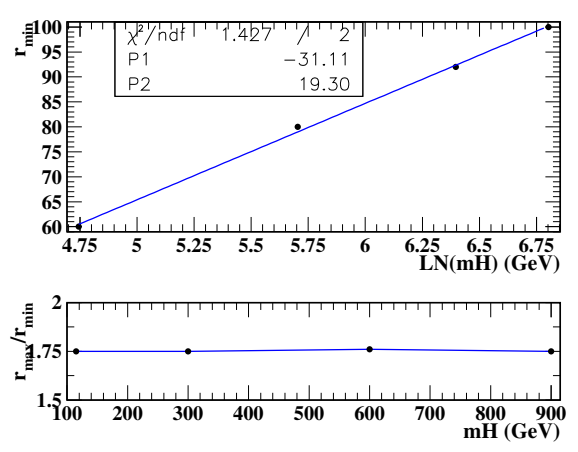

Figure 8: The minor radius and the ratio of the semi-diameters for the most probable region ellipses as a function of the Higgs mass.

ing fermion masses as $m_{u_{4}}=m_{e_{4}}=435 \mathrm{GeV}, m_{v_{4}}=365 \mathrm{GeV}$ and $m_{d_{4}}=455 \mathrm{GeV}$. For all the three open circles the mass of the heavier neutrino, $m_{N 4}$, was initially taken to be the same as its lighter partner. Keeping their equivalent Dirac mass constant, $\left(\sqrt{m_{v_{4}} \times m_{N_{4}}}=\right.$ const. $)$, their ratio $\left(m_{N_{4}} / m_{v_{4}}\right)$ was increased from 1 to higher values in steps of 0.5 alongside the solid line connecting the closed circles. The highest value of the ratio is fixed by the lowest experimentally allowed $v_{4}$ mass of $80 \mathrm{GeV}$. The plot shows that the increase of the ratio causes an initial decrease in $T$ which later becomes an increase. This behaviour is accompanied by a continuous slight decrease in $S$. The combination of these two has the potential of driving the test points into the allowed region, even for the $m_{H}=900 \mathrm{GeV}$ case, the farthest from the $S-T$ minimum. It can be concluded that the asymmetry between the two Majorana neutrino masses provides an opportunity for accommodating very heavy Higgs bosons in the Fourth Family model. While the allowed parameter space for the Dirac case has recently been considered narrow[30], it is clear that allowing neutrinos to be of Majorana-type significantly extends the range of possibilities. 


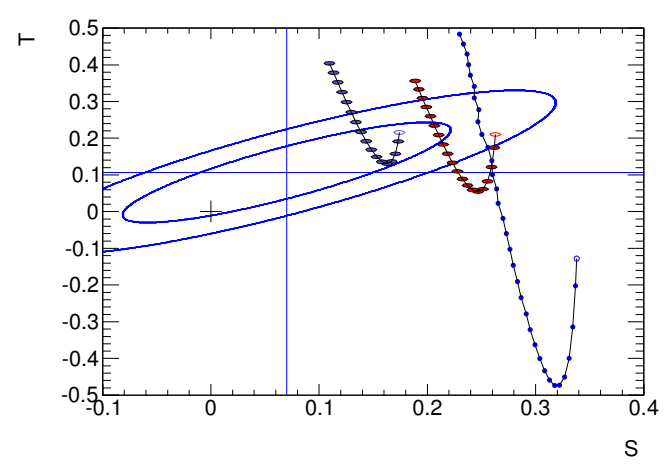

Figure 9: The impact of the Higgs boson and the mass ratio of the two Majorana neutrinos. For three example scenarios with different Higgs boson masses, the effect of changing the neutrino mass ratio is shown on the $S-T$ plane. In each case, the open circles represent degenerate Majorana neutrinos (ie. the equivalent of a single Dirac-type neutrino). The 1 and $2 \sigma$ error ellipses are from the 2009 results of the $U=0$ fit from LEP EWWG [37].

\section{The fourth SM family from Flavor Democracy}

Referring the reader to the original $[31,32,33]$ and to the review [34, 35, 36] papers for details, here we summarize the main FD results. The FD hypothesis leads the generic case of $n$ SM families to $n-1$ massless families and one super massive SM family with equal fermion masses. Given the measured masses of the third SM family fermions, FD requires the existence of the fourth SM family as the super massive one. Mass and mixing patterns of the first three family fermions are provided by small deviations from the full flavor democracy. It should be noted that FD consequences are independent on concrete mechanism of fermion's mass generation (e.g. Higgs mechanism), the sole requirement is that this mechanism should be the same for all SM fermions.

If the common Yukawa constant of the fermion-Higgs interactions is taken to be equal to $\mathrm{SU}_{W}(2)$ coupling constant $\mathrm{g}_{W}$, the fourth family fermion masses are equal to $2 \sqrt{2} g_{W} \eta$ (where $\eta \approx 245 \mathrm{GeV}$ ), therefore, $m_{4} \approx 4 \sqrt{2} m_{W} \approx 450 \mathrm{GeV}$. On the other hand, the fourth family masses are restricted from above $\left(m_{4}<1 \mathrm{TeV}\right)$ due to partial wave unitarity. Depending on the nature of the neutrino, again we consider two distinct cases:

- The fourth family neutrino is of Dirac nature. Then its mass should be equal to $m_{4}$, leading to $m_{d_{4}} \approx m_{e_{4}} \approx$ $m_{v_{4}} \approx m_{4}$. The Higgs boson's mass, and the size of the quark mixing between the fourth and the third generations are left as free parameters.

- The fourth family neutrino is of Majorana nature. In this case, the two Majorana particles have masses $m_{v_{4}} \approx$ $\left(m_{4}\right)^{2} / M$ and $m_{N_{4}} \approx M$, where $M$ is the Majorana mass scale. The current experimental lower bound $m_{v_{4}}>80 \mathrm{GeV}$ leads to $M<2500(6100) \mathrm{GeV}$ if $m_{4}=$ $450(700) \mathrm{GeV}$.

Another possible deviation is due to the large value of the $t$ quark mass: $u_{4}$ mass is expected to be somehow different than $m_{4}$. In the remaining of this section we will use OPUCEM to investigate the compatibility of these estimations with the 2009 EW precision data [37].

\subsection{SM4 with Dirac neutrinos}

For the scenario with Dirac-type neutrinos, we consider an example case with $m_{d_{4}}=m_{e_{4}}=m_{v_{4}}=m_{4}=550 \mathrm{GeV}$. The impact of increasing Higgs boson mass is shown in Figure 10 for various values of the sine of the mixing angle between the third and the fourth generations $\left(\sin \theta_{34}\right)$. The Higgs boson mass was scanned from $115 \mathrm{GeV}$ up to $415 \mathrm{GeV}$ in steps of $50 \mathrm{GeV}$. The $u_{4}$ mass is set to $610 \mathrm{GeV}$, compatible with the FD predictions and the findings of the previous section. It can be seen that a larger mixing angle increases $T$, compensating for larger Higgs boson mass values pulling towards lower $T$ and slightly higher $S$ values. The best cases are obtained for either small mixing angle (up to $\left|\sin \theta_{34}\right|=0.05$ ) and small Higgs mass (up to $150 \mathrm{GeV}$ ). Another possibility resulting in the $2 \sigma$ error ellipse is attained with a larger mixing angle ( $\left|\sin \theta_{34}\right|=0.09$ ) and a large Higgs mass (around $300 \mathrm{GeV}$ ).

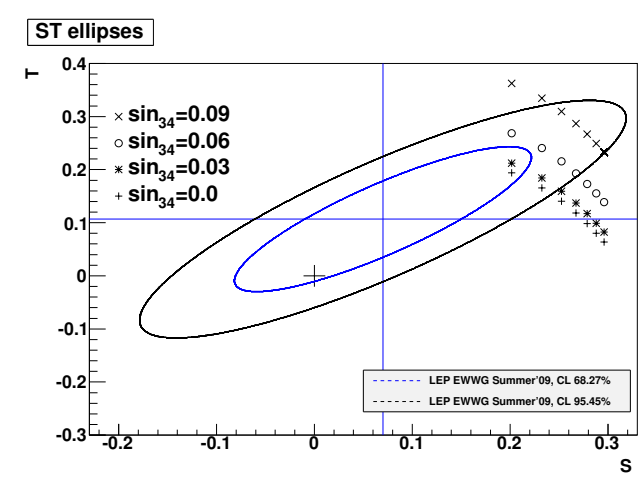

Figure 10: The Higgs mass dependence for various values of the third and fourth family quarks mixing angle, $\left|\sin \theta_{34}\right|$.

A similar approach was taken to study the impact of the neutrino mass, $m_{v_{4}}$, on the fourth family model's compatibility with the EW precision data. The Figure 11 contains a scan of the Higgs boson mass, from $115 \mathrm{GeV}$ up to $415 \mathrm{GeV}$ in steps of $50 \mathrm{GeV}$ for 5 different values of $m_{v 4}, 480,510,550,590$ and $620 \mathrm{GeV}$. The other relevant parameters are $m_{u_{4}}=610 \mathrm{GeV}$ and $m_{d_{4}}=m_{e_{4}}=550 \mathrm{GeV}$. The third and fourth family quarks mixing angle is set as $\left|\sin \theta_{34}\right|=0.03$. One can conclude that although $m_{v_{4}}=550 \mathrm{GeV}$ and $m_{H}=115 \mathrm{GeV}$ describe the parameters most suitable to EW data and also compatible with $\mathrm{FD}$, a change in the $v_{4}$ mass up to $\pm 70 \mathrm{GeV}$ is also allowed as long as the Higgs boson mass remains less than $215 \mathrm{GeV}$.

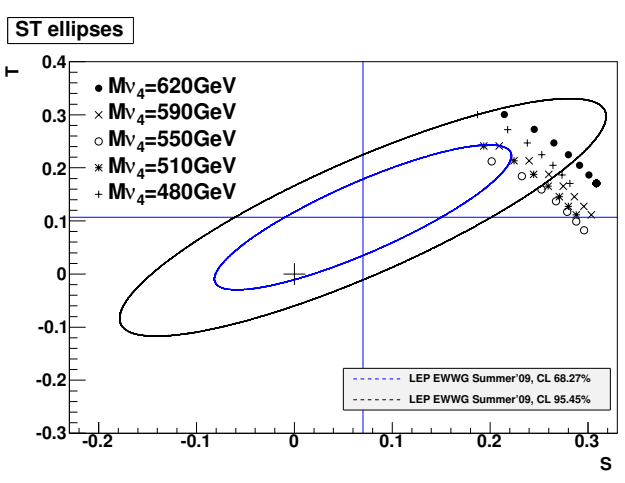

Figure 11: The Higgs mass dependence for various values of the fourth family lighter neutrino, $v_{4}$.

The impact of the change in $u_{4}$ mass is shown in Figure 12 for two Higgs boson mass values. The other relevant parameters are $m_{d_{4}}=m_{e_{4}}=m_{v_{4}}=550 \mathrm{GeV}$ and $\left|\sin \theta_{34}\right|=0.03$. The $u_{4}$ mass was scanned from 480 up to $630 \mathrm{GeV}$ in steps of $10 \mathrm{GeV}$. As the $u_{4}$ mass increases, $T$ value decreases and hits a minimum, after that $T$ keeps increasing, meanwhile $S$ decreases slowly but constantly. For larger values of the Higgs mass the same behaviour is observed with a starting point consistent with previous observations, i.e. an increase in $S$ and a decrease in $T$. One can conclude that although the $m_{H}=115$ $\mathrm{GeV}$ and $m_{u_{4}}=610 \mathrm{GeV}$ is most compatible with the EW fits, other $u_{4}-H$ mass pairs are also yielding results very close to 
$1 \sigma$ ellipse boundary; e.g. $m_{H}=115 \mathrm{GeV}, m_{u_{4}}=490 \mathrm{GeV}$ or $m_{H}=150 \mathrm{GeV}, m_{u_{4}}=610 \mathrm{GeV}$. It is interesting to note that the $T$ minimum is obtained for $m_{u_{4}}=m_{4}=550 \mathrm{GeV}$.

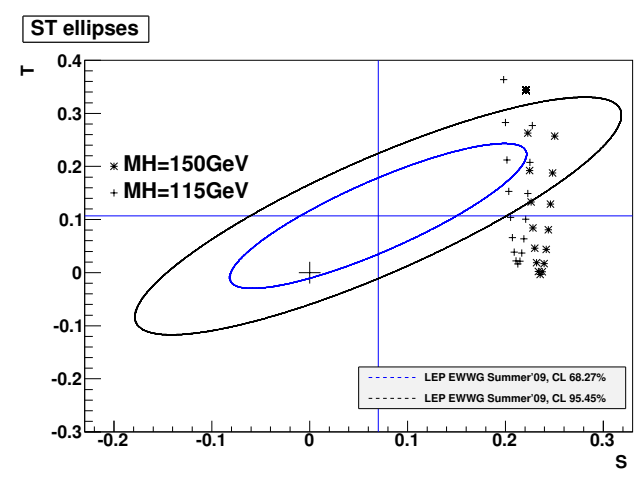

Figure 12: The fourth family up type quark, $u_{4}$ mass dependence for various values of the Higgs mass.

A cross check was performed by taking the two most compatible $u_{4}$ mass values found in the previous paragraph and the Higgs mass was increased from 115 up to $335 \mathrm{GeV}$ in steps of $20 \mathrm{GeV}$. The other parameters were kept same as before, namely $m_{d_{4}}=m_{e_{4}}=m_{v_{4}}=550 \mathrm{GeV}$ and $\left|\sin \theta_{34}\right|=0.03$. The results are shown in Figure 13. One can conclude that for $m_{u_{4}}=610(490) \mathrm{GeV}$ Higgs masses up to $215(175) \mathrm{GeV}$ give results within the $2 \sigma$ ellipse. However the smaller Higgs masses are favoured.

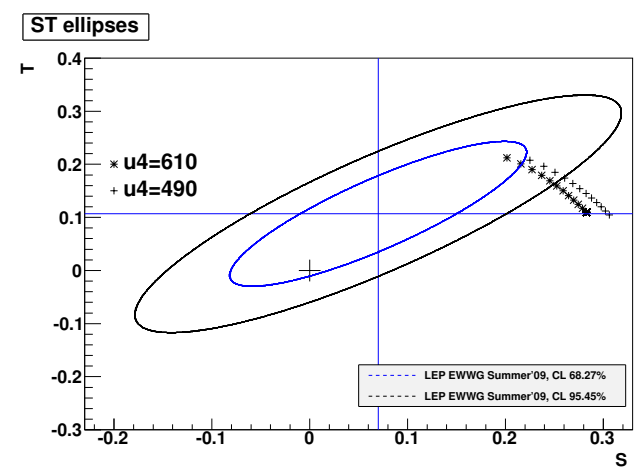

Figure 13: The Higgs boson mass dependence for various values of the fourth family up type quark, $u_{4}$.

The third and fourth families mixing angle dependence has also been investigated. The Figure 14, shows all the mass points within the $2 \sigma$ error ellipse resulting from a scan of $m_{u_{4}}$ and $\left|\sin \theta_{34}\right|$ values. The contributions from Higgs bosons with different mass values are shown using different markers. Other relevant parameters are kept as before, namely $m_{d_{4}}=m_{e_{4}}=m_{v_{4}}=550 \mathrm{GeV}$.

For $\left|\sin \theta_{34}\right|$ up to 0.07 , there are two allowed $u_{4}$ mass regions: one above and one below the $m_{4}$ mass. Inside these regions, smaller (larger) Higgs masses necessitate $u_{4}$ masses closer (farther) to the $m_{4}$ mass. With the increase of $\left|\sin \theta_{34}\right|$ value, the two regions merge at 0.07 and continue up to 0.17 , the maximum allowed value. One should also note that the $m_{u_{4}} \geq m_{4}$ case has a larger allowed range of parameters, it allows the highest values of the Higgs mass, its larger portion survives if the mass points are required to reside in the $1 \sigma$ error ellipse. Therefore according to FD, and also following the observed hierarchy of the second and third generations, one expects $m_{u_{4}}>m_{d_{4}}$.

Finally for different values of $m_{4}$, the free parameters of the Dirac case were scanned. The Table 1 contains the mass and mixing parameters for the best $\triangle \chi^{2}$ values obtained from a scan of all relevant parameters. It is seen that the favoured values are $m_{H}=115 \mathrm{GeV},\left|\sin \theta_{34}\right|=0.02$ and $m_{u_{4}}-m_{4}=$

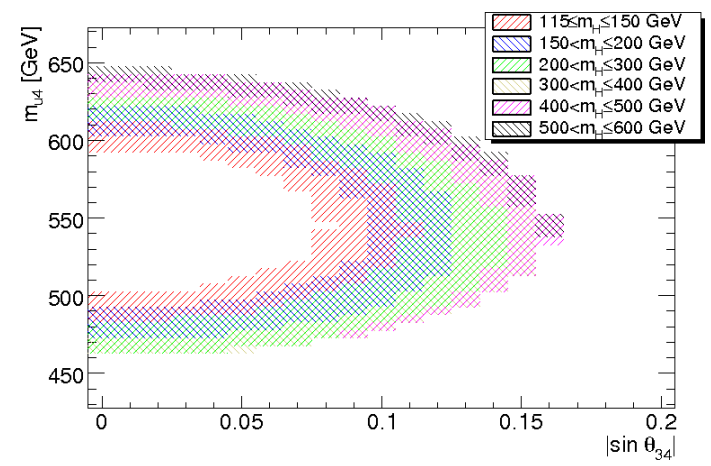

Figure 14: The $u_{4}$ vs $\left|\sin \theta_{34}\right|$ scan. The contributions from Higgs bosons with different mass values are shown in different colors.

$60 \mathrm{GeV}$, independent from $m_{4}$ mass. For all three $m_{4}$ values, we find that $\triangle \chi^{2}$ comparable to or lower than the 3-family SM's $\triangle \chi^{2}=1.7$ is achievable.

Table 1: The fourth family parameters at the best $\triangle \chi^{2}$ values compiled for FD hypothesis, Dirac scenario.

\begin{tabular}{c|c|c|c}
$m_{4}(\mathrm{GeV})$ & 400 & 550 & 700 \\
\hline$m_{u 4}(\mathrm{GeV})$ & 460 & 610 & 760 \\
\hline $\sin \theta_{34}$ & 0.03 & 0.02 & 0.02 \\
\hline$m_{H}(\mathrm{GeV})$ & 115 & 115 & 115 \\
\hline$\triangle \chi_{\min }^{2}$ & 1.58 & 1.67 & 1.72 \\
\hline$S$ & 0.198 & 0.202 & 0.204 \\
\hline$T$ & 0.202 & 0.202 & 0.208
\end{tabular}

\subsection{SM4 with Majorana neutrinos}

If the neutrinos are of Majorana type, an additional parameter, namely the mass of the heavier neutrino $\left(m_{N_{4}}\right)$ has to be taken into account. This section deals with this case using an approach similar to the previous section. The default mass pattern is set as: $m_{d_{4}}=m_{e_{4}}=m_{4}=550, m_{v_{4}}=135, m_{N_{4}}=$ $2500 \mathrm{GeV}$ and the Higgs mass greater than $115 \mathrm{GeV}$. In accordance with the FD predictions, the $v_{4}-N_{4}$ mass ratio is selected to yield a equivalent Dirac mass close to $m_{4}$ and the $u_{4}-d_{4}$ mass difference is constrained to be less than $m_{W}$. Figure 15 shows a scan of the Higgs boson mass from 150 up to $900 \mathrm{GeV}$ in steps of $50 \mathrm{GeV}$ for 3 different values of the mixing angle between the third and fourth generations. It can be seen that a larger mixing angle increases $\mathrm{T}$, compensating for larger Higgs boson mass values pulling towards higher $S$ and lower $T$ values. The best cases are obtained for either small mixing angle (up to $\left|\sin \theta_{34}\right|=0.05$ ) and Higgs mass between 150 and $350 \mathrm{GeV}$. If the mixing angle is increased up to $\left|\sin \theta_{34}\right|=0.1$, then a higher Higgs mass range of 550-600 $\mathrm{GeV}$ becomes feasible. 


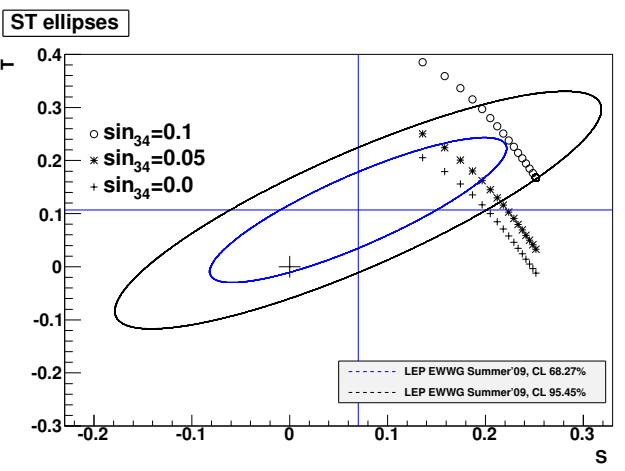

Figure 15: The Higgs mass dependence for various values of the third and fourth family quarks mixing angle, $\left|\sin \theta_{34}\right|$.

The Figure 16 displays the dependency on the $v_{4}$ and Higgs mass values. The Higgs boson mass was scanned from 150 up to $900 \mathrm{GeV}$ in steps of $50 \mathrm{GeV}$ for 4 different $v_{4}$ masses: $129,132,135$ and $138 \mathrm{GeV}$. Other relevant parameters are $\left|\sin \theta_{34}\right|=0.02, m_{u_{4}}=545 \mathrm{GeV}, m_{d_{4}}=m_{e_{4}}=550 \mathrm{GeV}$ and $m_{N_{4}}=2500 \mathrm{GeV}$. One can notice that the effect of increasing the $v_{4}$ mass is the opposite of increasing the mixing angle, $\left|\sin \theta_{34}\right|$ : it leads to smaller T values. Under the studied conditions, the most compatible values are obtained for $m_{v_{4}}=135 \mathrm{GeV}$ and $150<m_{H}<300 \mathrm{GeV}$.

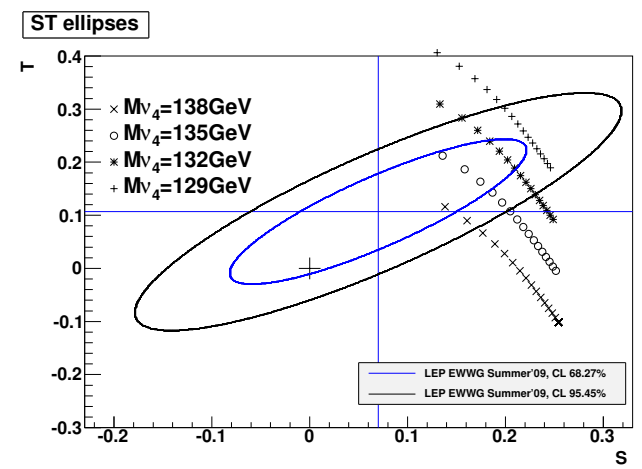

Figure 16: The Higgs mass dependence for various values of the fourth family lighter neutrino mass, $v_{4}$.

The Figure 17 displays the dependency on the $N_{4}$ and Higgs mass values. The $N_{4}$ mass was scanned from 1900 up to $4000 \mathrm{GeV}$ in steps of $100 \mathrm{GeV}$ for four Higgs boson masses: $115,150,200$ and $350 \mathrm{GeV}$. The other relevant parameters are $\left|\sin \theta_{34}\right|=0.02, m_{u_{4}}=545 \mathrm{GeV}, m_{d_{4}}=m_{e_{4}}=550 \mathrm{GeV}$ and $m_{v_{4}}=135 \mathrm{GeV}$. One can notice that the effect of increasing the $N_{4}$ mass is to decrease $T$ sharply and $S$ very mildly. Once more the most compatible results (closest to the EW precision fit minimum) are obtained for the lightest possible Higgs mass $(115 \mathrm{GeV})$ for $N_{4}$ mass between 2700 and $3300 \mathrm{GeV}$ but also the higher Higgs masses (e.g. $350 \mathrm{GeV}$ ) stays within the $1 \sigma$ error ellipse if the $N_{4}$ mass is between 2100 and $2300 \mathrm{GeV}$.

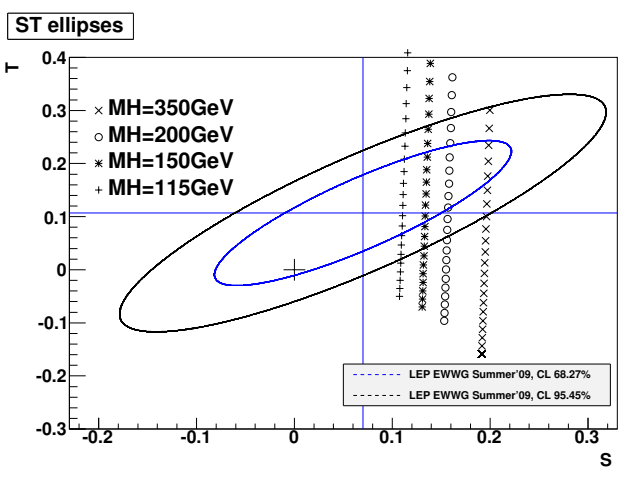

Figure 17: The fourth family heavier neutrino, $N_{4}$ mass dependence for various values of the Higgs mass.

Up to this point the $u_{4}-d_{4}$ mass difference was kept at $5 \mathrm{GeV}$. The impact of increasing this difference for different values of $v_{4}$ mass can be seen in Figure 18. The $u_{4}$ mass was scanned from 500 up to $580 \mathrm{GeV}$ in steps of $5 \mathrm{GeV}$ for three values of $v_{4}$ mass: $132,135,138 \mathrm{GeV}$. The other relevant parameters are $m_{H}=150 \mathrm{GeV}$ and $m_{N_{4}}=2800 \mathrm{GeV}$ (one of the best pairs found in the previous paragraph), $m_{d_{4}}=m_{e_{4}}=$ $550 \mathrm{GeV}$ and $\left|\sin \theta_{34}\right|=0.02$. The $u_{4}$ mass increase leads to an initial decrease of $T$ and after a minimum it results in an increase in $T$ while the $S$ values keep decreasing very slightly. For example the minimum for $m_{v_{4}}=135 \mathrm{GeV}$ occurs around $m_{u_{4}}=545 \mathrm{GeV}$ but any value of $u_{4}$ mass between 530 and $560 \mathrm{GeV}$ is rather close to the $\mathrm{EW}$ data fit minimum.

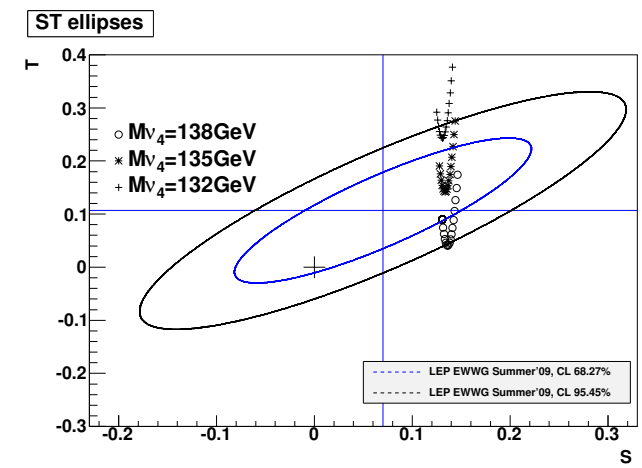

Figure 18: The fourth family up type quark, $u_{4}$ mass dependence for various values of the fourth family lighter neutrino mass, $v_{4}$.

To cross check the optimization of the heavier neutrinos mass, $m_{N_{4}}$, it was also scanned from 2500 up to $4000 \mathrm{GeV}$ in steps of $100 \mathrm{GeV}$ for two values of $u_{4}$ mass obtained in the previous paragraph. The Figure 19 shows the results of this scan. The other relevant parameters are $\left|\sin \theta_{34}\right|=0.02$, $m_{v_{4}}=135 \mathrm{GeV}, m_{d_{4}}=m_{e_{4}}=550 \mathrm{GeV}$ and $m_{H}=150 \mathrm{GeV}$. It can be seen that as in Figure 17, the $N_{4}$ mass which yields the most compatible result is $2800 \mathrm{GeV}$. 


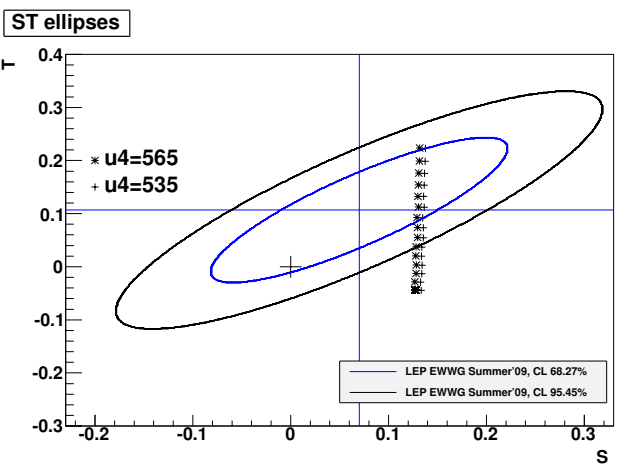

Figure 19: The fourth family up type quark, $u_{4}$ mass dependence for various values of the fourth family heavier neutrino, $N_{4}$.

Finally, for different values of $m_{4}$, all the free parameters of the Majorana case were scanned with the constraint $m_{\text {Dirac }} \equiv \sqrt{m_{N_{4}} \times m_{v_{4}}}=m_{4}$ while changing the $\underline{m}_{N_{4}}$ to $m_{v_{4}}$ ratio. The $u_{4}$ (Higgs) mass was scanned in steps of 5 (10) GeV, the mixing angle in steps of 0.01 and the $N_{4}$ to $v_{4}$ mass ratio in steps of 2 . The Table 2 contains the mass and mixing parameters for the best $\triangle \chi^{2}$ values obtained from a scan of all relevant parameters. It is seen that the favoured values are $m_{H}=115 \mathrm{GeV},\left|\sin \theta_{34}\right|=0.02, m_{N_{4}} / m_{v_{4}}=14$ and $m_{u_{4}}-m_{4}=50 \mathrm{GeV}$, independent of $m_{4}$ mass. We note that the minimum $\triangle \chi^{2}$ obtained with the scan is much lower than the 3-family SM's $\triangle \chi^{2}=1.7$ for all three $m_{4}$ values.

Table 2: The fourth family parameters at the best $\triangle \chi^{2}$ values compiled for FD hypothesis, Majorana scenario.

\begin{tabular}{c|c|c|c}
$m_{4}(\mathrm{GeV})$ & 400 & 550 & 700 \\
\hline$m_{u_{4}}(\mathrm{GeV})$ & 450 & 600 & 750 \\
\hline$m_{N_{4}}(\mathrm{GeV})$ & 1497 & 2058 & 2618 \\
\hline$m_{v_{4}}(\mathrm{GeV})$ & 107 & 147 & 187 \\
\hline$\left|\sin \theta_{34}\right|$ & 0.02 & 0.02 & 0.02 \\
\hline$m_{H}(\mathrm{GeV})$ & 115 & 115 & 115 \\
\hline$\triangle \chi_{\min }^{2}$ & 0.143 & 0.187 & 0.215 \\
\hline$S$ & 0.110 & 0.115 & 0.117 \\
\hline$T$ & 0.136 & 0.139 & 0.146
\end{tabular}

\section{Conclusions}

We have presented OPUCEM, an open-source function library publicly available to calculate the electroweak oblique parameters $S, T$ and $U$ for a number of physics models. This library has been heavily tested and shown to be able reproduce various earlier results successfully. It provides safe-to-use functions with an error-checking machinery that relies on comparisons amongst different formulas and between exact calculations and well-known approximations. The current version is expected to compile, run and give correct results using any Unix-like platform with the C++ Technical Report 1 (TR1) compiler support (e.g. g++ 4.2 or later).

OPUCEM is meant to set an example to a new concept that we call "open-formula" papers. Many scientific papers include numerical examples of various symbolic calculations. With time, such examples loose a lot of their value, unless they are not readily reproducible by others. Reproduction of old results is usually very difficult, since most representations of the numerical results are on plots and figures, which provide only limited precision when one wants to check an independent reimplementation of the symbolic calculations. As a solution to this, we suggest authors of scientific papers, which present and demonstrate new formulas, commit the actual computer code of their numerical calculations on a publicly available repository. This will also help resolve errors that occasionally happen when the results are sent to publication, such as misprinting of the formulas.

In addition to the library itself, we have also presented an example of its use in studying the mass parameters of a fourth generation of Standard Model fermions. Investigations yielded regions of the parameter space favored by the precision EW data, both for Dirac and Majorana cases of the fourth SM family neutrinos. For the Dirac-type neutrinos, the allowed mass parameters describe an elliptical ring in the $m_{u_{4}}-m_{d_{4}}$, $m_{v_{4}}-m_{e_{4}}$ plane when the sum of the fourth generation quark masses and the sum of the fourth generation lepton masses are kept constant. The radius and the thickness of this elliptical ring are dependent on the mass of the Higgs boson and the mixing angle between the third and fourth generation quarks. We also note that on this ring, EW data favours $m_{u_{4}}>m_{d_{4}}$ and $m_{v_{4}}<m_{e_{4}}$. For the Majorana case, the additional parameter (the mass of the heavier neutrino) gives more flexibility to the choice of parameters and especially by adjusting the ratio of the masses of the new neutrinos, it is possible to move some additional portions of the parameter space to become compatible with the measured $S, T$ values. Finally, we test predictions of the Flavor Democracy hypothesis and find that in either Majorana or Dirac case, for the FD predictions to be true, the sine of the mixing angle should be about $0.02, m_{u_{4}}$ should be about 50-60 GeV higher than the generic 4th generation mass scale and the smaller values of the Higgs mass are preferred. Under these conditions, the $\Delta \chi^{2}$ measured on the $S, T$ plane for an FD-motivated 4-generation SM is lower than the 3-generation SM.

\section{References}

[1] D.C. Kennedy. and B. W. Lynn, Nucl. Phys. B 322 (1989) 154.

1

[2] M. E. Peskin and T. Takeuchi, Phys. Rev. D 46 (1992) 381. 1

[3] A. Kundu and P. Roy, Int. J. Mod. Phys. A 12 (1997) 1511. 1

[4] G. Bhattacharyya, S. Banerjee and P. Roy, Phys. Rev. D 45, 3 (1992) R740. 1

[5] S. Dawson and C.B. Jackson, Phys. Rrev. D 76 (2007) 015014. 1

[6] B.A. Kniehl and H.-G. Kohrs, Phys. Rev. D 48 (1993) 225. 1, 2, 2.1, 1

[7] OPUCEM project at CEDAR Hepforge repository, http://projects.hepforge.org/opucem/ 1

[8] B. Holdom et al., PMC Phys. A (2009) 3:4 2, 3

[9] Beyond the 3SM, 1st workshop at CERN, http://indico.cern. ch/conferenceDisplay. py? conf $I d=33285.2,3$

[10] Beyond the 3SM, 2nd workshop in Taiwan, http://indico.cern. ch/conferenceDisplay. py? conf $I d=68036.2,3$

[11] GNU Compiler Collection, http://gcc.gnu.org/ 2

[12] Cygwin project, http://www.cygwin.com/ 2

[13] MinGW project, http://www.mingw.org/ 2 
[14] S.L. Moshier, http://www.moshier.net/ 2

[15] H.-J. He, N. Polonsky and S. Su, Phys. Rev. D 64 (2001) 053004. 2

[16] E. Gates, J. Terning, Phys. Rev. Lett. 67 (1991) 1840. 2

[17] Chanowitz, Phys. Rev. D 79 (2009) 113008 [arXiv:0904.3570]. 2, 3, 3.1, 4

[18] J.R. Forshaw, D.A. Ross, B.E. White, JHEP 0110:007 (2001) [arXiv:hep-ph/0107232]. 2

[19] A.A. Natale, P.S. Rodriges Da Silva, Mod. Phys. Lett. A10 (1995) 1829. 2

[20] G. D. Kribs et al., Phys. Rev. D 76 (2007) 07516. 2.2, 2

[21] I. Antcheva et al., Comput. Phys. Commun. 180, 12 (2009) 2499. 2.2, 2.3

[22] MONO project, http://www.mono-project.com/ Main_Page 2.3

[23] GAMBAS project, http://gambas.sourceforge. net/en/main.html 2.3

[24] QtCreator page, http://qt.nokia.com/products/ developer-tools 2.3

[25] V. A. Novikov, L. B. Okun, A. N. Rozanov and M. I. Vysotsky, Phys. Lett. B 529, 111 (2002) [arXiv:hepph/0111028]; V. A. Novikov, L. B. Okun, A. N. Rozanov and M. I. Vysotsky, JETP Lett. 76, 127 (2002) [arXiv:hep-ph/0203132]. 3

[26] M. Hashimoto, Phys. Rev. D 81 (2010) 075023 [arXiv:1001.4335]. 3.2

[27] O. Eberhardt, A. Lenz and J. Rohrwild, arXiv:1005.3505v2 (2010). 3.2

[28] LEP Electroweak Working Group summer 2006 results web page, http://lepewwg.web.cern.ch/LEPEWWG/ plots/summer2006/s06_stu_contours.eps 3.2

[29] C. Amsler et al., Phys. Lett. B 667 (2008) 1. 3.2

[30] J. Erler and P. Langacker, arXiv:1003.3211v2 (2010). 3.3

[31] H. Fritzsch, Phys. Lett. B 289, 92 (1992). 4

[32] A. Datta, Pramana 40, L503 (1993). 4

[33] A. Celikel, A. Ciftci and S. Sultansoy, Phys. Lett. B 342, 257 (1995). 4

[34] S. Sultansoy, Why the Four SM Families (2000), http: //cdsweb.cern.ch/record/436180 4

[35] S. Sultansoy, AIP Conf. Proc. 899, 49 (2007) [arXiv:hepph/0610279]. 4

[36] S. Sultansoy, Int. Conf. Part. Phys.: In memoriam Engin Arik and her colleagues, Istanbul, Turkey, 27-31 Oct 2008 [arXiv:0905.2874]. 4

[37] LEP Electroweak Working Group summer 2009 results ( $S=0.05 \pm 0.10, T=0.10 \pm 0.09$, correlation coefficient $\rho=0.85$ ) obtained through private communication. We shift these results to take into account the following reference masses: $m_{H}^{r e f}=115 \mathrm{GeV}, m_{t}^{r e f}=173.1 \mathrm{GeV}$.

9,4 